\title{
Nonspecific Alpha-Naphtyl Acetate Esterase Activity of T-Lymphocytes: Study in Healthy Newborns and Children, in Immune Deficiencies and Juvenile Rheumatoid Arthritis
}

\author{
NORIYUKI WADA, ANNE-MARIE PRIEUR, ${ }^{(16)}$ AND CLAUDE GRISCELLI \\ Groupe de Recherches d'Immunologie et de Rheumatologie Pédiatriques, INSERM U 132, Département de Pédiatrie, \\ Hôpital des Enfants Malades, Paris, France
}

\begin{abstract}
Summary
The aim of this study was to compare the $E$ rosette-forming cells and nonspecific alpha naphtyl esterase (ANAE)-positive lymphocyte values in normal and pathologic situations. In newborns, the ANAE-positive lymphocytes represented less than $60 \%$ of the $E$ rosette population. During the first year of life, $E$ rosetteforming cells (E-RFC) reached normal values as soon as one month whereas only three-fourths of the $T$ cells exhibited an ANAE-positive staining. In adult $T$ cell populations, nearly $90 \%$ were ANAE-positive. Our observations of immune deficiencies suggested that the relative proportions of E-RFC and ANAEpositive lymphocytes were generally comparable to normal values. However, in the majority of the patients with very low or absent E-RFC (severe combined immune deficiencies, Di George syndrome, and congenital rubella), some ANAE-positive lymphocytes could be detected. Our immunologic survey shows that the ANAEpositive lymphotytes were in a normal range 2 years after a bone marrow transplantation in severe combined immune deficiencies patients. One child who exhibited a normal amount of E-RFC and whose lymphocytes failed to respond in vitro to mitogens had practically no ANAE-positive lymphocytes. An elevated amount of ANAE-positive cells in juvenile rheumatoid arthritis may reflect an augmentation of the $T$ helper functions which permanently stimulated in vivo immunoglobulin production.
\end{abstract}

\section{Speculation}

The alpha-napthtyl acetate esterase-positive lymphocytes have been shown to belong to the $T$ helper subpopulation. Various proportions of these cells among $T$-lymphocytes can be expected in normal subjects such as newborns and infants where the immune system is considered as immature and in situations involving immune regulation such as immune deficiencies and chronic hyperimmune diseases.

The histochemical staining for nonspecific alpha naphtyl esterase (ANAE) has been used to characterize subpopulations of human lymphocytes and monocytes $(9,12)$. The staining pattern is clearly distinctive between monocytes which exhibit a diffuse red brown cytoplasm esterase reaction and lymphocytes which contain one to three esterase-positive granules adjacent to the cell membrane. The ANAE-positive lymphocytes have been shown to belong to the $T$ cell subpopulation where it represents about $90 \%$ of the $\mathrm{E}$ rosette-forming cells (E-RFC) fraction in normal human peripheral blood (9). These esterase-positive lymphocytes are characteristic of $\mathrm{T}$ lymphocyters with receptors for the Fc portion of $\operatorname{IgM}(\mathrm{T} \mu)$ whereas the remaining esterase-negative $\mathrm{T}$ cells have receptors for the $\mathrm{Fc}$ portion of $\operatorname{IgG}(\mathrm{T} \gamma)(6)$. It has also been suggested that $\mathrm{T} \gamma$ and $\mathrm{T} \mu$ have different in vitro functional activities and could exert opposing effects on $\mathrm{B}$ cell response to pokeweed mitogen, $\mathrm{T} \mu$ helping the $\mathrm{B}$ cell differentiation, $\mathrm{T} \gamma$ suppressing this phenomenon (13).

The aim of this study was to compare the $\mathrm{E}$ rosette-forming cells and ANAE-positive lymphocyte values in normal and pathologic situations. We found that ANAE-positive lymphocytes are significantly lowered at birth, but reach adult values after one year of life. In pathologic conditions, the most striking observations were the presence in active juvenile rheumatoid arthritis of a high level of ANAE-positive lymphocytes which could signify an excess of "helper" function and a low number of ANAE-politive lymphocytes in some immune deficiencies which could be related to a reduction of helper function.

\section{MATERIALS AND METHODS}

Four groups of children were studied.

(1) Twenty-seven children with primary immunodeficiency diseases met the World Health Organization criteria (3): seven severe combined immunodeficiencies, one $\mathrm{X}$-linked agammaglobulinemia (X-AG), six "common variable" hypogammaglobulinemia, one complete and three incomplete Di George syndromes, six ataxia telangiectasia, one Wiskott-Aldrich, one intestinal lymphangiectasia, and one unclassified immunodeficiency.

(2) Thirteen children had various diseases in which immune abnormalities may be suspected: three congenital rubella, two myasthenia, two lymphohistiocytosis, two severe denutritions, and four polymorphonuclear dysfunctions.

(3) A group of children had inflammatory diseases: 20 juvenile rheumatoid arthritis (JRA), two systemic lupus erythematosus, one peri-arteritis nodosa, and nine miscellaneous chronic inflammatory states.

(4) Twelve young patients had infections: two staphylococcus infections, two bacterial sepsis, five chronic candidiasis, one virus infection, one tuberculosis, and one lepra. Controls were 20 young normal adults, 20 children hospitalized for various diseases without any immunologic or inflammatory symptoms, and 37 infants ( 15 normal newborns and 22 infants between 1 and 12 months of age).

Heparinized peripheral blood samples were obtained from patients and controls, and the lymphocyte-rich fraction was obtained after Ficoll triosil centrifugation. The $\mathrm{E}$ rosette formation was performed by incubating $1.16^{6} / \mathrm{ml}$ of the mononuclear cells with $150 \mu \mathrm{l}$ of a $2 \%$ sheep red blood cells suspension $\left(30 \mathrm{~min}\right.$ at $37^{\circ} \mathrm{C}$ ), and then centrifuged for $5 \mathrm{~min}\left(200 \times g\right.$ at $\left.4^{\circ} \mathrm{C}\right)$, and stored overnight at $4^{\circ} \mathrm{C}$ and the percentage of E-RFC evaluated (11).

ANAE activity in the mononuclear cell fraction was tested according to Horwitz et al. (9). Two hundred $\mu$ l of $1.10^{6}$ cells $/ \mathrm{ml}$ 
were pelleted onto slides by cytocentrifugation, dried, and immediately fixed in $4^{\circ} \mathrm{C}$ buffered formalin acetone. The esterase reaction was performed with freshly prepared $\alpha$-naphtyl acetate solution mixed with hexazotized pararosanilin for $60 \mathrm{~min}$ at room temperature. The slides were rinsed free of visible deposits and counterstained with $0.5 \%$ toluidin blue solution. The monocytes displayed a diffuse, cytoplasmic, red brown ANAE positive staining, and a fraction of the lymphocyte exhibited one to three red brown cytoplasmic granules adjacent to the cell membrane. B lymphocytes were identified by surface immunoglobulin as previously described (11).

\section{RESULTS}

COMPARATIVE PERCENTAGES OF E ROSETTES AND ANAE-POSITIVE LYMPHOCYTES IN NORMAL SUBJECTS AT VARIOUS AGES

As shown on Table 1, the percentages of the granular staining lymphocytes were contstantly lower than the percentages of ERFC. The percentages of newborn E-RFC and ANAE-positive lymphocytes were significantly lower than those observed at one month $(P<0.02)$. Furthermore, in newborn, the ANAE-positive lymphocytes represented less than $60 \%$ of the $E$ rosette population. During the first year of life, E-RFC reached normal values as soon as one month whereas only three-fourths of the $T$ cells exhibited an ANAE-positive staining. In adult $T$ cell populations, nearly $90 \%$ were ANAE positive.

Table 1. Comparison between the percentages of E-RFC and ANAE-positive lymphocytes in newborns, children, and adults

\begin{tabular}{lcccc}
\hline \multicolumn{1}{c}{ Ages } & $\begin{array}{c}\text { No. } \\
\text { tested }\end{array}$ & $\begin{array}{c}\text { E-RFC } \\
(\%)\end{array}$ & $\begin{array}{c}\text { ANAE }+1 y^{1} \\
(\%)\end{array}$ & $\begin{array}{c}\text { \% of } \\
\text { ANAE + ly } \\
\text { among } \\
\text { E-RFC }\end{array}$ \\
\hline Newborn & 15 & $46.7 \pm 18.3^{2}$ & $27.8 \pm 6.7^{3}$ & 58 \\
1 month & 7 & $62.5 \pm 12.4^{4}$ & $48.4 \pm 12.1^{5}$ & 77 \\
2-12 months & 15 & $56.4 \pm 10.4^{4}$ & $44.2 \pm 6.1^{5}$ & 78 \\
1-15 years & 20 & $63.0 \pm 10.2^{4}$ & $53.6 \pm 9.4^{6}$ & 84 \\
Adult & 20 & $59.9 \pm 13.2^{4}$ & $54.2 \pm 10.5^{6}$ & 91 \\
\hline
\end{tabular}

' ANAE + ly = lymphocytes displaying granular cytoplasmic nonspecific esterase staining.

${ }^{2-6} 2$ compared to ${ }^{4}, P<0.02 ;{ }^{3}$ compared to ${ }^{5}, P<0.01 ;{ }^{5}$ compared to ', $P<0.002$.
COMPARATIVE PERCENTAGES OF E-RFC AND ANAE-POSITIVE LYMPHOCYTES IN SEVERE COMBINED IMMUNE DEFFICIENCIES (SCID)

Seven patients with SCID were tested (Table 2). Four of them belonged to the category of SCID with a selective defect of precursor T lymphocytes (6) (R. G., G. N., and A. S.) and three were associated with an adenosine deaminase deficiency (S. J., T. E., and B. M.). Three of four patients tested before transplantation had a notable number of ANAE-positive cells (mean $=13 \%$ ) even in the total absence of E-RFC cells (T. E.). The fourth child (F. M.) who had a graft-versus-host (GVH) disease due to a maternofoetal transfusion verified by karyotype study and HLA typing, exhibited a low percentage of blood $\mathrm{T}$ cells among which about $50 \%$ were ANAE-positive.

Three patients who were fully reconstituted after bone marrow transplantation had normal values of E-RFC (mean $=66.3 \%$ ) and ANAE-positive lymphocytes (mean $=52 \%$ ), 2 years or more after transplantation. One patient had normal values of E-RFC but still a low percentage of ANAE-positive cells 10 months after a bone marrow transplantation (T. E.).

COMPARATIVE PERCENTAGES OF E-RFC AND ANAE-POSITIVE LYMPHOCYTES IN VARIOUS IMMUNEDEFICIENCIES

As seen in Table 3, in one patient with a complete Di George syndrome (N. B.) who had no T lymphocyte which formed $E$ rosettes, the percentage of ANAE-positive lymphocytes was also very small. In three patients with incomplete Di George syndrome (with characteristic cardiac malformation and hypoparathyroidism), the percentages of both E-RFC and ANAE-positive cells were reduced. However, in the case where E-RFC were very low (Z. G.), the number of ANAE-positive cells was higher than the E-RFC. In the other $T$ cell deficiencies tested (Wiskott-Aldrich syndrome, ataxia telangiectasia, and intestinal lymphangiectasia), the percentages of ANAE-positive lymphocytes were moderately reduced, but proportions of ANAE-positive cells among E-RFC were within normal values. No important abnormality was found in common variable hypogammaglobulinemia except in two cases: R. R., who had a percentage of ANAE-positive cells higher than the E-RFC, and R. M., who had a very low percentage of ANAEpositive lymphocytes. The most striking abnormality was observed in a patient with an unclassified immune deficiencies (D. L.) whose $\mathrm{T}$ lymphocytes did not exhibit esterase activity while able to form $\mathrm{E}$ rosettes.

Table 2. E-RFC and ANAE-positive lymphocytes in SCID before and after transplantation

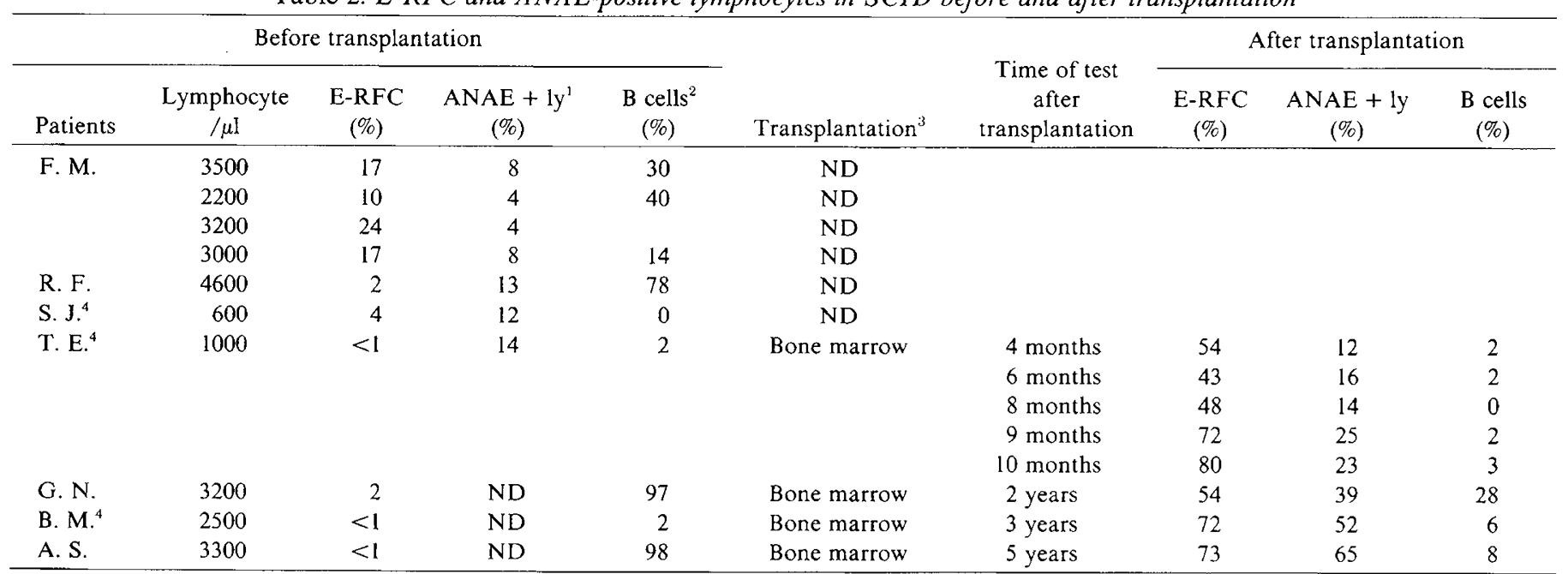

'ANAE + ly = lymphocytes displaying granular cytoplasmic nonspecific esterase staining.

${ }^{2}$ Determined by surface immunoglobulin markers.

${ }^{3}$ The lymphocyte absolute numbers were in normal range after bone marrow tarnsplantation.

${ }^{4}$ Adenosine deaminase-negative SCID. 
Table 3. Comparison between the percentage of E-RFC and the percentage of ANAE-positive lymphocytes in various immune deficiencies

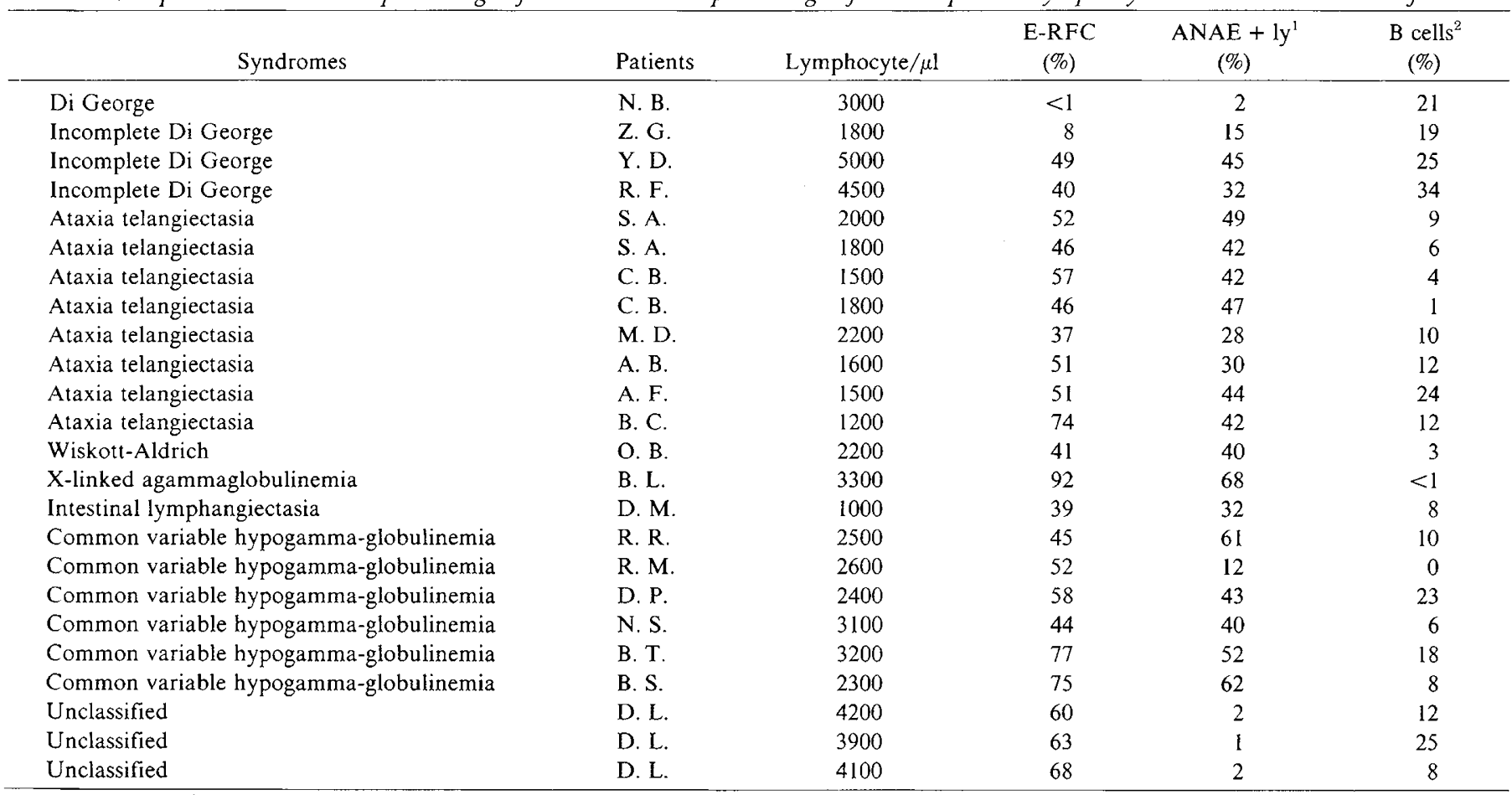

${ }^{\prime} \mathrm{ANAE}+\mathrm{ly}=$ lymphocyte dysplaying granular cytoplasmic non specific esterase staining.

${ }^{2}$ Determined by surface immunoglobulin markers.

\section{COMPARATIVE PERCENTAGES OF E-RFC AND ANAE-POSITIVE} LYMPHOCYTES IN MISCELLANEOUS DISEASES

We studied various pathologic situations where the immune system can be impaired. No major abnormality was found in myasthenia, lymphohistiocytosis, or severe malnutrition. In two cases of congenital rubella with decreased percentage of E-RFC, the ANAE-positive subpopulations represented about $70 \%$ of the $E$ rosetting cells. In the third case of congenital rubella, although no $T$ cells were detectable with the E rosette technique, $35 \%$ of the lymphocytes appeared to be ANAE-positive. In four cases with a granulocyte abnormality and frequent severe infections, the $E$ RFC and the ANAE-positive lymphocytes were normal.

COMPARATIVE PERCENTAGES OF E-RFC AND ANAE-POSITIVE LYMPHOCYTES IN INFLAMMATORY DISEASES

Figure 1 represents the percentage of ANAE-positive lymphocytes among the E-RFC in normal children $(A)$ and adults $(B)$. The average values found in inflammatory diseases $(C)$ and infections $(D)$ are similar to those found in normal children and adults. However, four patients among these two subgroups had elevated values. The percentage of E-rosettes in these four cases was low $(38,32,45$, and $16 \%$, respectively), whereas the average percentage of ANAE-positive cells was normal. One of these patients had a chronic inflammatory disease with rash fever and lympho-adenopathy, one patient had an hyperimmunoglobulin $\mathrm{E}$ syndrome (2), and the two others chronic candidiasis. One patient with systemic lupus erythematous in group $\mathrm{C}$ had only $30 \%$ of ANAE-positive cells among her E-RFC. In JRA patients $(E)$, it was found that the ANAE-positive cells always were more numerous than the E-RFC, and ANAE-positive granule appeared to be voluminous and more intensively coloured in some patients with active disease.

\section{DISCUSSION}

ANAE activity had previously been shown to correlate with the sheep red blood cell rosette-forming cells in human peripheral blood and described as a useful $T$ cell marker $(9,12)$. In our studies, the procedure prescribed by Horwitz et al. (9) for ANAE staining was strictly followed and ANAE-positive lymphocytes seemed to correlate strongly with the $\mathrm{T}$-cell population because $5.3 \pm 2.8 \%$ of E-RFC and $5 \pm 2.8 \%$ of ANAE-positive lymphocytes were found in a E-RFC depleted population (12 experiments). In

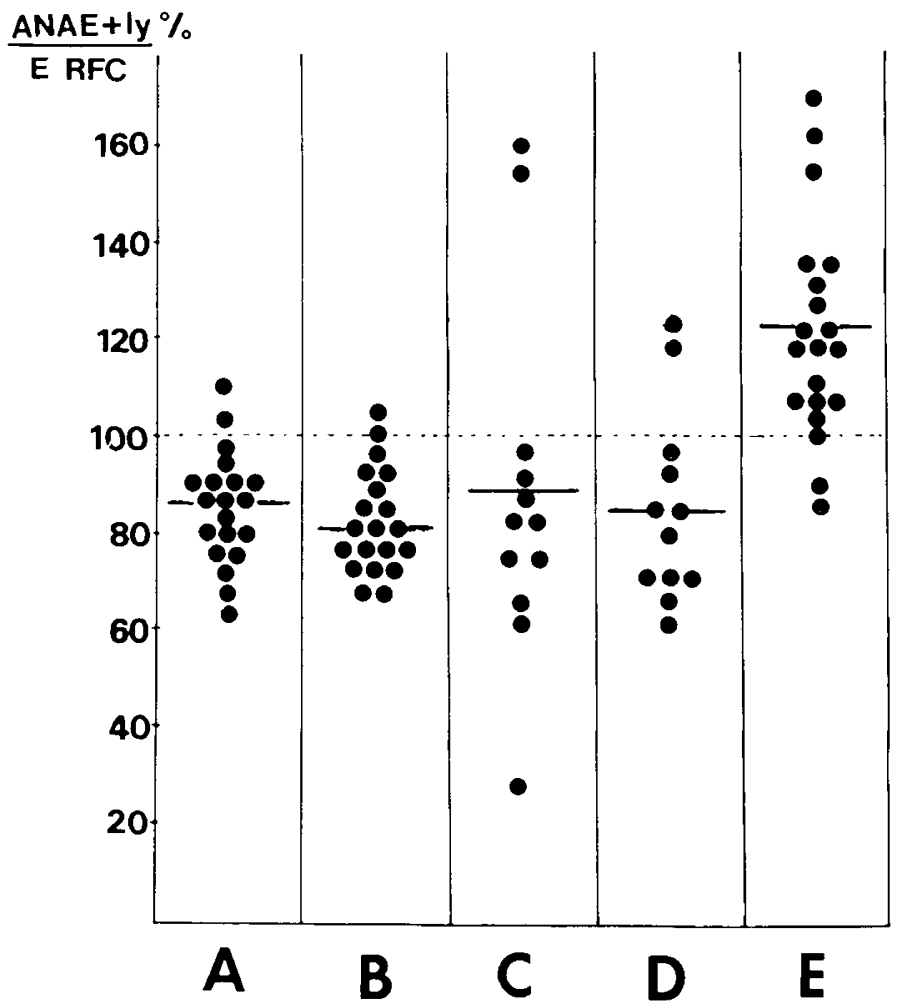

FIG. 1. 
T-cell, ANAE appeared as granular staining pattern easily distinguishable from the diffuse cytoplasmic esterase reaction which characterized monocytes $(9,12)$. Our study confirmed that in the newborn the percentage of E-rosettes was significantly lower than that present at one month of age (7) and established that more than $40 \%$ of $\mathrm{T}$ cells were ANAE negative. These two $\mathrm{T}$ cell markers increased during the first month, but the ANAE-positive cells remained at a significant lower level during the first year of life. Later, the percentage of ANAE-positive lymphocytes reached about $90 \%$ of the E-RFC, a value in agreement with previous reports (9).

Our observations in immune deficiencies suggested that the relative proportions of E-RFC and ANAE-positive lymphocytes was generally comparable to the normal. However, in the majority of the patients with very low or absent E-RFC (SCID, Di George syndrome, and congenital rubella), some ANAE-positive lymphocytes could be detected. This is peculiarly striking in three SCID where nearly $13 \%$ of the ANAE-positive cells had a typical granular aspect which cannot be confused with the monocyte type esterase staining. (If weak ANAE staining exists in the monocytes of the patients, this could mimic ANAE-positive lymphocytes). The possibility that immature B cells can contain ANAE-positive granules must be considered, but in the patient R. F. whose $B$ cells were elevated, there was no overlapping between the percentage of B lymphocytes and the percentage of ANAE-positive cells. This esterase-positive fraction of lymphocytes probably belongs to the $\mathrm{T}$ subpopulation which has only a low capacity of $\mathrm{E}$ rosetting. This hypothesis is supported by the fact that in two SCID patients (F. M. and S. J.), the number of T lymphocytes detected using an heterologous anti-human thymocyte were higher (31 and 20\%, respectively) than ANAE-positive and E-rosette lymphocytes.

Our immunologic survey shows that the ANAE-positive lymphocytes were in a normal range 2 years after a bone marrow transplantation in SCID patients. Within the first months after engraftment, the percentage of ANAE-positive cells remained at the same level as before transplant whereas the E-RFC gradually increased (patient T. E.). Because cell marker studies indicated that the transplanted cells reconstituted the $T$ cell compartment (5), we suspected that the donor precursor T lymphocytes had not yet undergone a full maturation (this could take several months). Another explanation could be that this phenomenon was related to the mild GVH reaction consistently observed after a bone marrow transplantation; in another SCID patient who had GVH due to maternal-fetus transfusion, the donor $\mathrm{T}$ lymphocytes had only a poor esterase activity.

On the other hand, no major abnormality was detected in the other immunedeficiencies in which percentage of E-RFC was within or closed the normal range. Normal values were also found in diseases in which an immunologic dysfunction is suspected such as myasthenia, severe malnutrition, and lymphohystiocytosis. However, one child who exhibited a normal amount of E-RFC and whose lymphocytes failed to respond in vitro to mitogens had nearly no ANAE-positive lymphocytes. His lymphocytes strongly resembled immature T cells; these are ANAE negative as are the mouse cortical thymocytes (14), a subpopulation of human thymocytes and human lymphocyte transformed in vitro by exposure to polyclonal mitogens (12).

Among chronic inflammatory diseases, e.g., JRA, we found almost constantly that a greater number of ANAE-positive lymphocytes than of E-RFC. This is in agreement with Knowles et al. (12) who reported $8 \%$ of ANAE-positive cells in the E-RFC depleted fraction in one patient with rheumatoid arthritis (12). This discrepancy between the percentages of $\mathrm{E}$ rosettes and ANAE-positive lymphocytes was more frequently observed in patients with very active systemic type of the disease (1). In these cases, the intracytoplasmic granules sometimes appeared to be much bigger and intensively coloured than did those of normal lymphocytes. Whether this granular staining reflects a peculiar aspect of the monocyte in chronic inflammatory diseases is un- known, but in this case, one would have expected a similar profile in other chronic inflammatory conditions. Furthermore, in three patients with JRA, the study was performed before and after nylon wool column adherent cell depletion; the numbers of ANAE-positive lymphocytes were not modified, whereas the cells exhibiting a diffuse ANAE staining were removed. On the other hand, the mean of E-RFC in JRA was slightly reduced (49\% \pm 16.4), and the average number of ANAE-positive lymphocytes was higher $(60 \% \pm 12)$ than that of the age-matched controls in the same age group. In fact, the absolute number of mononuclear cells in patients was significantly increased, whereas the $T$ cell compartment appeared to be of normal size (10). Our findings in JRA appeared to be restricted to this disease; it was not observed in systemic lupus erythematosus.

The ANAE-positive $T$ lymphocytes in normals have been shown to correlate with the $T$ cells which have receptors for the Fc portion of $\operatorname{IgM}(\mathrm{T} \mu)(16)$. These $\mathrm{T} \mu$ lymphocytes are able to help B cell proliferation and differentiation into plasma cells in response to pokeweed mitogen (PWM), whereas the ANAE-negative lymphocytes correspond to the $T$ cells with $F c$ receptor for IgG $(\mathrm{T} \gamma)(\mathrm{T} \gamma$ cells suppress B cell differentiation in the presence of PWM (13)). Our present finding of an elevated amount of ANAE-positive cells in JRA may reflect an augmentation of the $\mathrm{T}$ helper functions which permanently stimulated in vivo immunoglobulin production. This observation is complementary to that of the possibility of a serum factor in JRA which could inhibit the suppressive cells (15). This is also in agreement with the observation that the E-rosette positive, ANAE-negative lymphocytes of patient $D$. L. do not contain $T \mu$ subpopulation and were unable to help normal B cells to differenciate into plasma cells in the presence of PWM (data not shown).

The decrease in the ANAE-positive lymphocyte subpopulation in newborn does not correlate with $T \mu$ values which are comparable to those observed in adult (7) and which had been shown to exert a suppressive activity on B cells maturation $(4,8)$. Thus, the significance of the relatively low number of esterase positive lymphocytes in newborn remains unexplained.

\section{REFERENCES AND NOTES}

1. Ansell, B. M.: Heberden Oratio 1977: chronic arthritis in childhood. Ann. Rheum Dis., 37: 107 (1978).

2. Buckley, R. H., Wray, B. B., and Belmaker, E. Z.: Extreme hyperimmunoglobulinemia $\mathrm{E}$ and undue susceptibility to infection. Pediatrics, 49: 59 (1972).

3. Cooper, M. D., Fauld, W. P., Fudenberg, H. H., Good, R. A., Hitzig. W., Kunkel, H., Rosen. F., Seligmann, M., and Wedgwood, R. J.: Classification of primary immunodeficiencies. N. Engl. J. Med.. 288: 966 (1973).

4. Durandy, A., Fischer, A., and Griscelli, C.: Active suppression of B lymphocyte maturation by two different newborn T lymphocyte subsets. J. Immunol., I23: 2644 (1979).

5. Griscelli, C., Durandy, A., Ballet, J. J., Prieur, A.-M., and Hors, J.: T- and B-cell chimerism in two patients with severe combined immunodeficiency (SCID) after transplantation. Transplant. Proc., 9: 171 (1977).

6. Grossi, C. E.. Webb, S. R., Zicca, A., Lydyard, P. M., Moretta, L., Mingari, M. C., and Cooper, M. D.: Morphological and histochemical analyses of two human T-cell subpopulations bearing receptors for IgM or IgG. J. Exp. Med.. 147: 1405 (1978)

7. Gupta, S., and Good, R. A.: Subpopulations of human T-lymphocytes. III Distribution and quantitation in peripheral blood, cord blood, tonsils, bone marrow, thymus, lymph node, and spleen. Cell. Immunol., 36: 263 (1978).

8. Hayward, A. R., and Lydyard, M. M.: Suppression of B-lymphocyte differentiation of newborn T-lymphocytes with an Fc receptor for IgM. Clin. Exp. Immunol.. 34: 374 (1978).

9. Horwitz, D. A., Allison, A. C., Ward, P., and Kight, N.: Identification of human mononuclear leucocyte populations by esterase staining. Clin. Exp. Immunol., 30: 289 (1977)

10. Hoyeraal, H. M.. Froland, S. S., and Wisloff, F.: Lymphocyte populations and cellular immune reactions in juvenile rheumatoid arthritis. Scand. J. Immunol., 4: 801 (1975).

11. Jondal, M.. Wigzell, H., and Aiuti. F.: Human lymphocyte subpopulations: classification according to surface markers and/or functional characteristics. Transplant. Rev., 16: 163 (1973).

12. Knowles, D. M., Hoffman, H. T., Ferrarini, M., and Kunkel, H. G.: The demonstration of $\alpha$-naphtyl esterase activity in human lymphocytes: usefulness as a T-cell marker. Cell. Immunol., 35: 112 (1978).

13. Moretta, L., Webb, S. R., Gross, C. E., Lydyard, P. M., and Cooper, M. D.: Functional analysis of two human $\mathrm{T}$-cell subpopulations: help and suppression 
of B-cell responses by $\mathrm{T}$-cells bearing receptors for IgM or IgG. J. Exp. Med., 146: 184 (1977).

14. Mueller. J., Brun del Re. G., Buerki, H., Keller. H. U., Hess, M. N., and Cottier. H.: Non specific acid esterase activity: a criterion for differentiation of $T$ and B-lymphocytes in mouse lymph nodes. Eur. J. Immunol., 5: 270 (1975).

15. Strelkauskas. A. J.. Callery, R. T., Mc Donell, J., Borel, Y., and Schlossman, S.

T.: Direct evidence for loss of human suppressor cells during active autorm-

Copyright (C) 1981 International Pediatric Research Foundation. Inc $0031-3998 / 81 / 1509-1266 \$ 02.00 / 0$ mune disease. Proc. Natl. Acad. Sci. U. S. A., 75: 5150 (1978).

16. Requests for reprints should be addressed to: Anne-Marie Prieur, INSERM U 132. Hôpital des Enfants Malades, 149 rue de Sèvres, 75730 Paris Cedex 15, France.

17. This research was supported by grants from the INSERM (CRL-79-5-232-4)

18. Received for publication August 26, 1980.

19. Accepted for publication February 2, 1981 